\title{
Comprehensive Software Simulation on Ground Power Supply for Launch Pads and Processing Facilities at NASA Kennedy Space Center
}

\author{
JESUS A. DOMINGUEZ1', ELIAS VICTOR ${ }^{2}$, \\ ANGEL L. VASQUEZ ${ }^{3}$, ALFREDO R. URBINA ${ }^{2}$ \\ ${ }^{1}$ Vencor-ESC, Kennedy Space Center, Florida 32899 USA \\ ${ }^{2}$ NASA, Kennedy Space Center, Florida 32899 USA \\ 3 Jacobs, Kennedy Space Center, Florida 32899 USA \\ CORRESPONDING AUTHOR: JESUS A. DOMINGUEZ (jesus.a.dominguez@nasa.gov)
}

\begin{abstract}
A multi-threaded software application has been developed in-house by the Ground Special Power (GSP) team at NASA Kennedy Space Center (KSC) to separately simulate and fully emulate all units that supply VDC power and battery-based power backup to multiple KSC launch ground support systems for NASA Space Launch Systems (SLS) rocket. The software application was built using Java programming language in Microsoft Windows 8 Operating System (OS) to emulate the operation, communication, and functionality of each VDC-power supply and battery backup units concurrently in communication via Ethernet opensocket protocol with Allen-Bradley (AB) RSlogix-5000 Programming Logic Controllers (PLC) currently operated as redundant data acquisition and control system at SLS launch pads and processing facility sites. The software application also simulates all the input/output (IO) signals acquired and generated in the SLS field via Mimic ${ }^{\circledR}$, a software platform commercially available to build simulation on IO signals for RSlogix5000 PLC. All individual Java-based VDC-power unit simulators are concurrently integrated with the Mimic ${ }^{\circledR}$-based field IO simulation via Modbus communication protocol allowing a comprehensive simulation on GSP for SLS launch pads and processing facilities at NASA KSC.
\end{abstract}

INDEX TERMS Simulation, emulation, modeling, Space Launch Systems (SLS), Voltage Direct Current (VDC) power, Programmer Logic Controller (PLC), RSlogix-5000, Mimic ${ }^{\circledR}$, input/output (IO) signals, Java, multithread software, Modbus, Ground Special Power (GSP).

\section{INTRODUCTION}

GSP operative tasks in SLS ${ }^{[1][2]}$ include Mobile Launcher (ML), Launch Pad B (PADB), Multi-Payload Processing Facility (MPPF), Space Vehicle System, Boosters Control Power Distribution Unit (BCPDU), Actuator Control Unit (ACU), Trust Vector Control (TVC), Power Distribution Control Unit (PDCU), Battery Unit Interface Enclosure (BUIE), and Orion's Power Distribution Unit (PDU). The number of VDC-power supply and battery-backup units required by these GSP operative tasks at KSC varies widely from 32 units (24 power suppliers and 8 battery-backup units) required by Space Vehicle System to just 4 units ( 3 power suppliers and 1 battery-backup units) required by MPFF. Testing and verifying PLC code development in GSP redundant $\mathrm{AB}$ control systems require the implementation of a number of individual software simulation instances running in parallel and fully emulating not only the concurrent communication of the VDC-power supply and battery-backup units with both redundant PLCs but also the intrinsic operational features of the units such as, voltage setting, current load, operation status, fault detection, and trip triggering conditions. Some VDC-power supply and backup units are problematic as they are not standard off-the-shelf products but rather custom-built units with long span delivery designed and built by the unit's manufacturer to meet specific requirements unique for a given GSP operative task at NASA KSC. A single software application per unit manufacturer was developed in-house using Java multi- 
thread programming via NetBeans $\operatorname{IDE}^{[4]}$ software development platform in Windows 8 OS to conduct simulation and fully emulation of all VDC-power supply and battery-backup units utilized in a number of KSC GSP operative tasks for SLS rocket. Instead of waiting for the often-delayed delivery and installation of the actual units, Java-based simulation instances accurately emulate all the units in the field running autonomously and establishing communication in parallel via Ethernet open-socket protocol with both redundant AB RSlogix-5000 PLCs ${ }^{[3]}$.

NASA KSC GSP team conducted further software development work to implement a comprehensive simulator that emulates the entire GSP field operative tasks and includes not just VDC-power supply and battery-backup units but also all Remote Input/Output (RIO) signals. This comprehensive software simulator proved to significantly facilitate the development of AB RSlogix-5000 PLC control systems, the PLC's code review, customer demonstration, comprehensive application testing, virtual field operation, and operator training. To simulate the entire GSP operative field is necessary to emulate also all input/output (IO) signals generated in the field and acquired by PLC's RIO modules. Mimic $^{\circledR[5]}$, a commercially available simulation builder for AB RSlogix-5000 controllers, was used to emulate IO signals acquired and generated in the field. Mimic ${ }^{\circledR}$ cannot emulate data-transfer and command instructions that are transmitted between the VDC-power supply and batterybackup units and the $A B$ RSlogix-5000 controller via the $A B$ Eweb $^{[6]}$ module that is mounted in the RIO chassis. The Eweb module provides open-socket communications via Ethernet TCP/UDP links with all VDC power supplier and battery-backup units. The GSP Java-based simulation application deploys a number of stand-alone instances that fully emulate the same number of VDC power supplier and battery-backup units. As actual field units all simulation instances, one instance per VDC-power unit, are in communication with the $\mathrm{AB}$ Eweb module via Ethernet open-socket protocol.

In all KSC GSP operative tasks, VDC power is supplied in voltage-constant mode, the VDC power supply units are set by the operator leading to an electrical current value that depends on the total current load required by the VDCoperated devices present and activated in the field. As Javabased VDC-power supply unit simulators are not aware of the electrical current load simulated and deployed in Mimic ${ }^{\circledR}$, the GSP team implemented Modbus ${ }^{[7]}$ communication protocol in both Mimic ${ }^{\circledR}$ and Java-based VDC-power unit simulators to transmit the current load simulated in $\mathrm{Mimic}^{\circledR}$ to the VDC-power unit simulators upgrading in real time the electrical current value at the VDC-power unit simulators. Coupling both simulators based on $\mathrm{Mimic}^{\circledR}$ and Java programming via Modbus communication led to the implementation of a comprehensive software simulation on VDC ground power supply for SLS launch pads and processing facilities at NASA KSC.
The comprehensive software simulation application designed and implemented by the GSP team has been used to successfully emulate, so far, a total of nine different KSC GSP operative tasks that include Mobil Launcher (ML), Launch Pad B (PADB), Multi-Payload Processing Facility (MPPF), Boosters Control Power Distribution Unit (BCPDU), Actuator Control Unit (ACU), Trust Vector Control (TVC), Power Distribution Control Unit (PDCU), Battery Unit Interface Enclosure (BUIE), and Orion's Power Distribution Unit (PDU).

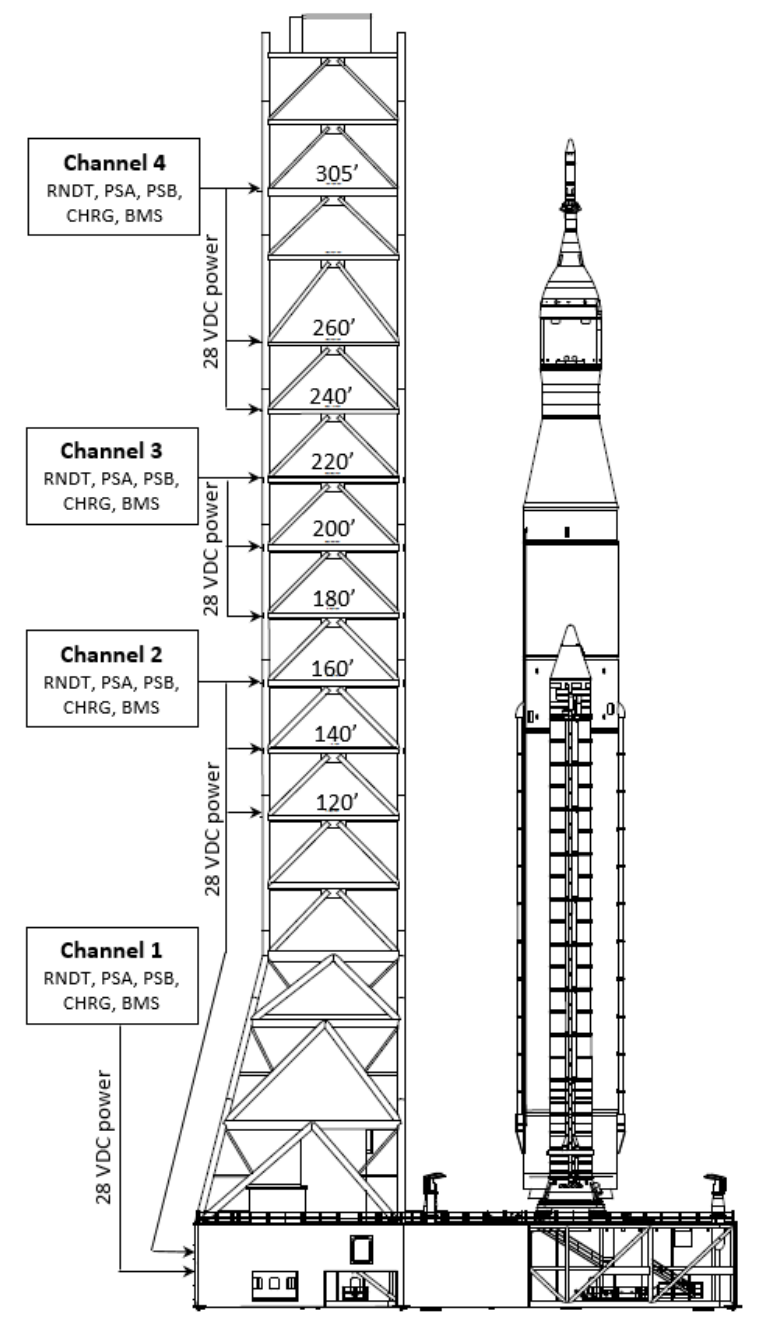

Figure 1. Layout of DC-power supply for ML system

Figure 1 depicts the layout of VDC-power supply for ML system consisting of four channels or VDC supply service lines at four different ML's tower elevations, starting at 0 feet (channel 1) and ending at 305' (channel 4). Each of one of the four channels is equipped with five VDC-power supply units, Redundant (RNDT), Charger (CHRG), Power System A (PSA), Power System B (PSB) and Battery Management System (BMS).

Using actual or scaled-down hardware to assess future development or upgrade current GSP systems is extremely 
difficult, time consuming, and very expensive. The implementation of a virtual environment via simulation would be a feasible alternative at much lower cost and much less time consuming as the control system can be virtually replicated to accommodate and evaluate additional hardware with less difficulty.

\section{SIMULATION DEVELOPMENT}

The GSP team built two independent but coupled simulators to implement and deploy a comprehensive GSP simulation application. One simulator, built using Java programming, emulates all VDC-power supply and batterybackup units in communication with the $\mathrm{AB}$ Eweb module via open-socket protocol. The second simulator, built using Mimic $^{\circledR}$ (a simulation building platform commercially available for AB RSlogix-5000 controllers) emulates field IO signals generated and acquired by AB RSlogix-5000 IO modules.

Mimic $^{\circledR}$ has access to the IO modules of the AB RSlogix5000 controller to emulate IO signals, but it does not have the capability of emulating Ethernet client-server opensocket data transfer between the $\mathrm{AB}$ Eweb module and the VDC-power units.

Ethernet Modbus communication capability was implemented in both simulators to couple them allowing electrical current load emulated by $\mathrm{Mimic}^{\circledR}$-based simulator to be transferred to the respective Java-based VDC-power supply unit simulator and been able to refresh unit's electrical current reading.

\section{A. SIMULATION ON VDC-POWER SUPPLY UNITS}

As Mimic $^{\circledR}$ cannot be used to emulate data-transfer from/to the AB Eweb module, the KSC GSP team designed, implemented, and deployed a multi-threaded software simulation application in Java programing language that fully emulates the units that supply VDC power, including battery-based power backups, for different KSC ground operative tasks at launch pads and processing facilities.

The Java-based simulator emulates not only actual Ethernet client-server open-socket communication of the VDC-power supply unit with the AB Eweb module, but also all relevant unit operation features including but not limited to handling of IO commands and generation of anomalous operational events, such as trip triggering, fault detection, and unit malfunction.

Different instances launched by the same GSP in-housebuilt Java-based simulator program emulate equal number of VDC-power supply units. Each instance emulating a given VDC-power supply unit, has different IP address and port number to communicate via open-socket Ethernet architecture with the Eweb modules of redundant systems A and B. The simulator emulates all built-in operative

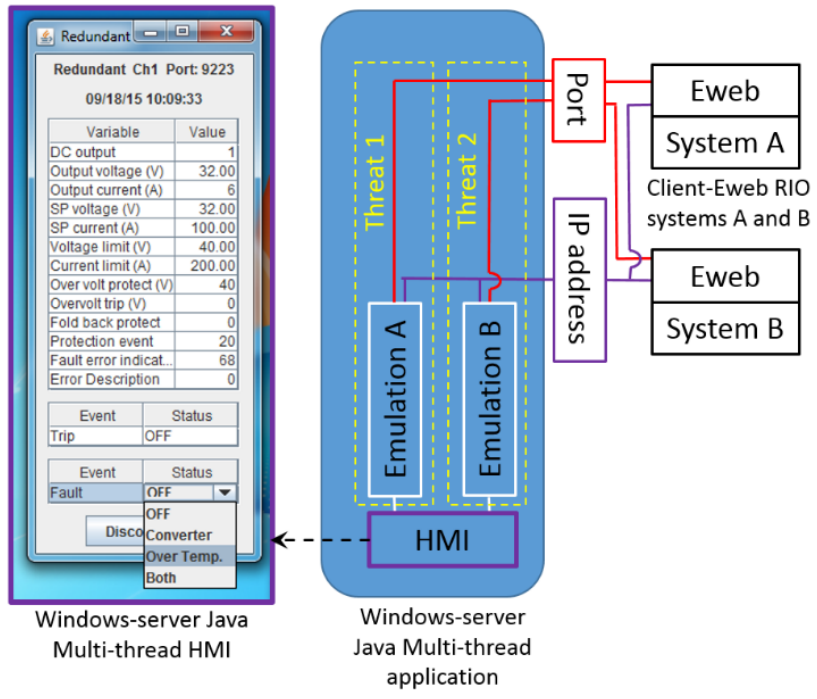

Figure 2. Schematic of Java-implemented multi-thread software to simulate DC-power units built by Ametek Power Instruments and used in GSP systems.

functions of actual VDC-power supply units used in the field.

Figure 2 illustrates the schematic of the Java-based simulator of a VDC-power supply unit manufactured by Ametek Power Instruments. Instances of the same Java software simulation application are launched to simulate individual VDC-power supply units having specific IP address and port number to communicate via client-server open-socket ENET architecture with both Eweb modules located in two redundant LCDs, systems A and B. The simulator emulates all built-in operative functions of a VDCpower supply unit manufactured by Ametek and includes trigger and detection of hardware faults.

The Simulated power unit also emulates the communication protocol of the actual Ametek VDC-power supply units. It uses a single port to communicate via open socket protocol with the Eweb modules in systems A and B. The multi-thread-based simulator also establishes opensocket communication with the Eweb module just as the actual VDC-power supply units. The application launches as many thread instances as needed, in parallel, to concurrently handle simulation tasks, such as simulator-Eweb data exchange, simulator's HMI in Windows OS, VDC-power supply operation, and detection of hardware faults.

A second Java-based multi-threaded software simulation application was designed, implemented, and deployed to simulate a special battery backup unit manufactured by Navitas and known as Battery Management System (BMS).

The BMS unit uses two dedicated ports, rather than one (as in the Ametek case) and the same IP address to communicate via open-socket protocol with the Eweb modules in redundant systems A and B. As in the Ametek case, the multi-thread-based simulator also establishes open- 
socket communication with the Eweb module just as the actual Navitas BMS unit does.

Figure 3 illustrates the schematic layout Java-based simulator of Navitas BMS. The simulator launches as many thread instances as needed, in parallel, to concurrently handle

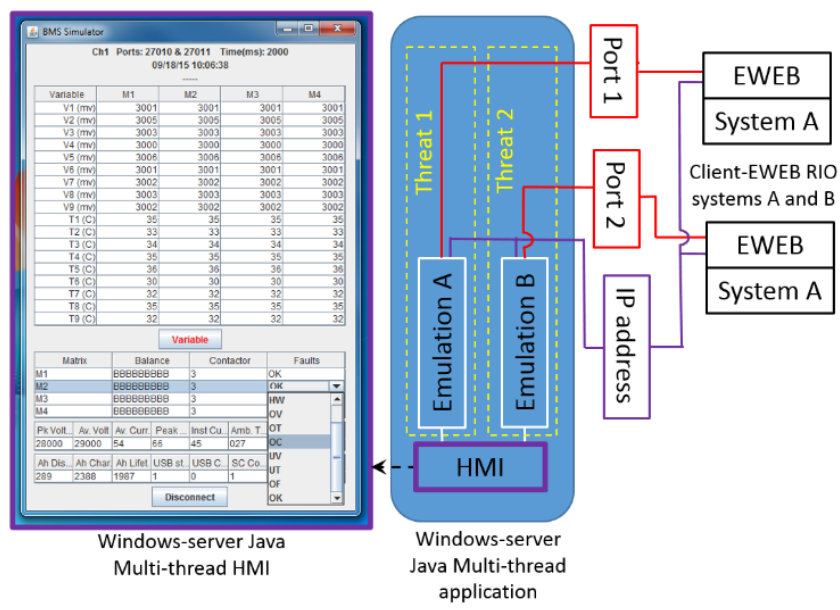

Figure 3. Schematic of Java-implemented multi-thread software to simulate Battery Management System (BMS) built by Navitas.

simulations tasks, such as simulator-Eweb data exchange, simulator's HMI in Windows OS, BMS unit operation, and triggering and detection of hardware faults.

\section{B. SIMULATION ON IO FIELD SIGNALS}

Mimic $^{\circledR}$, a Windows-based simulation enabler tool developed by MYNAH Technologies for AB RSlogix 5000 controllers, was used to emulate digital and analog IO signals acquired and generated in $\mathrm{AB}$ IO modules. $\mathrm{Mimic}^{\circledR}$ is organized in configuration layers. It provides an IEC-1131 based graphical block view of the simulated process, allowing users to configure models, download them into the runtime engine, and view the model data flow in real-time.

Key IO signals used in GSP systems and emulated by Mimic $^{\circledR}$ include both analog and digital types, such as voltage drop, electrical current load, motorized and manual breakers, and contactors connected to the VDC-power units that share and load their charge to the main power buses.

As stated above, Mimic ${ }^{\circledR}$ has access to the IO signals acquired and generated in the IO modules of the $A B$ RSlogix-5000 controller allowing their emulation and implementation of IO field signals, but it does not have the capability of emulating messages and data transfer via opensocket protocol in the $\mathrm{AB}$ Eweb module specifically used to communicate with the VDC-power supply units.

The Mimic $^{\circledR}$ capability of performing Modbus communication was utilized to transfer electrical current load value emulated and set in Mimic ${ }^{\circledR}$ simulator to the Javabased VDC-power supply simulator that supplies the respective electrical current load. Under Modbus protocol mode, Mimic ${ }^{\circledR}$ simulator uploads the emulated electrical current load value in a memory register, the Java-based simulator reads and downloads in real time the value to upgrade the value currently posted in the emulated VDCpower supply unit.

Figure 4 shows a snapshot of the HMI built to display Mimic ${ }^{\circledR}$ simulation on setting electrical loads in channel 2 of GSP PADB system through a motorized circuit breaker (CB205A) and a set of manual circuit breakers (CB200A2, CB200A3, CB200A4, and CB225A). Voltage and electrical current values displayed at the HMI of Figure 4 come from four in-house Java-based simulation instances emulating three different VDC-power supply units (PSA, RDNT, and CHGR) as illustrated in Figure 2 and one battery (BMS) respectively as illustrated in Fig 3. HMI's snapshot of Figure 4 also displays a set of emulated relay indicators for 2 load bar buses, (PSA and RDNT buses) and 2 battery taps (high and low taps).

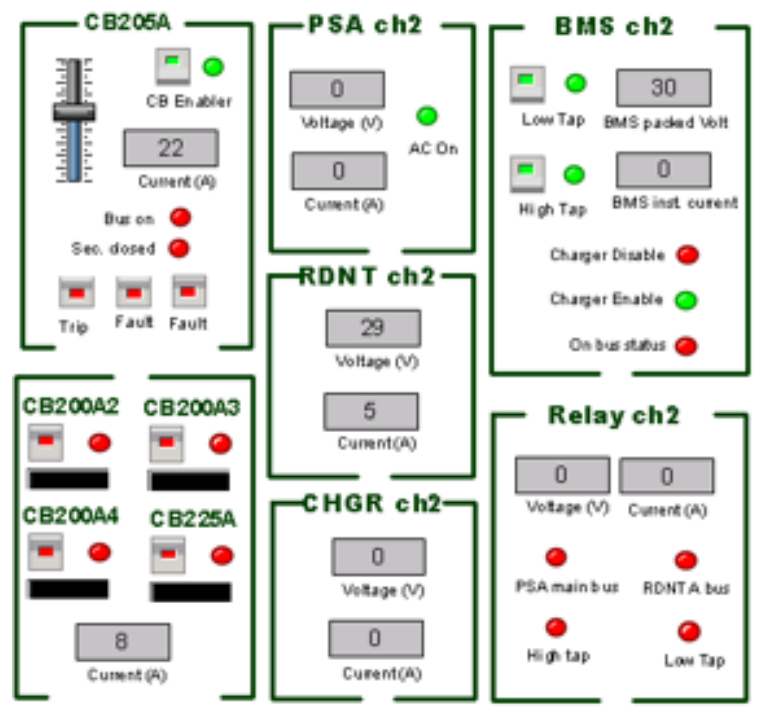

Figure 4. Mimic ${ }^{\circledR}$ HMI built to display the simulation on setting electrical loads through their respective circuit breakers.

\section{SIMULATION FUNCTIONAL APPLICATION}

This section highlights the functional aspect of the simulation software application used to emulate PADB, one of the nine GSP operative systems emulated by the software application.

\section{A. PADB OPERATIVE SYSTEM REVIEW}

As depicted in Figure 5, PADB operative task supplies VDC power at three different subsystems (also labeled as channels) at SLS launch Pad B built at KSC for heavy-lift launch of NASA's Orion spacecraft. Pad Terminal Connection Room (PTCR) is located in channel 1, liquid hydrogen $\left(\mathrm{LH}_{2}\right)$ storage tank and liquid oxygen $\left(\mathrm{LO}_{2}\right)$ storage tank are located in channels 2 and 3 respectively. PTCR is located on the east side of the water tank (see figure 5) underneath the elevated hardstand of the launch pad B. It 
is covered with as much as 20 feet of dirt fill. The liquid $\mathrm{H}_{2}$ and $\mathrm{O}_{2}$ tanks are located in opposite sides of launch pad $\mathrm{B}$, Figure 5 shows the $\mathrm{LH}_{2}$ storage tank. Ground VDC power is supplied to Pad B via these three channels with specific tasks, each channel is equipped with four power suppliers and one battery-backup unit.

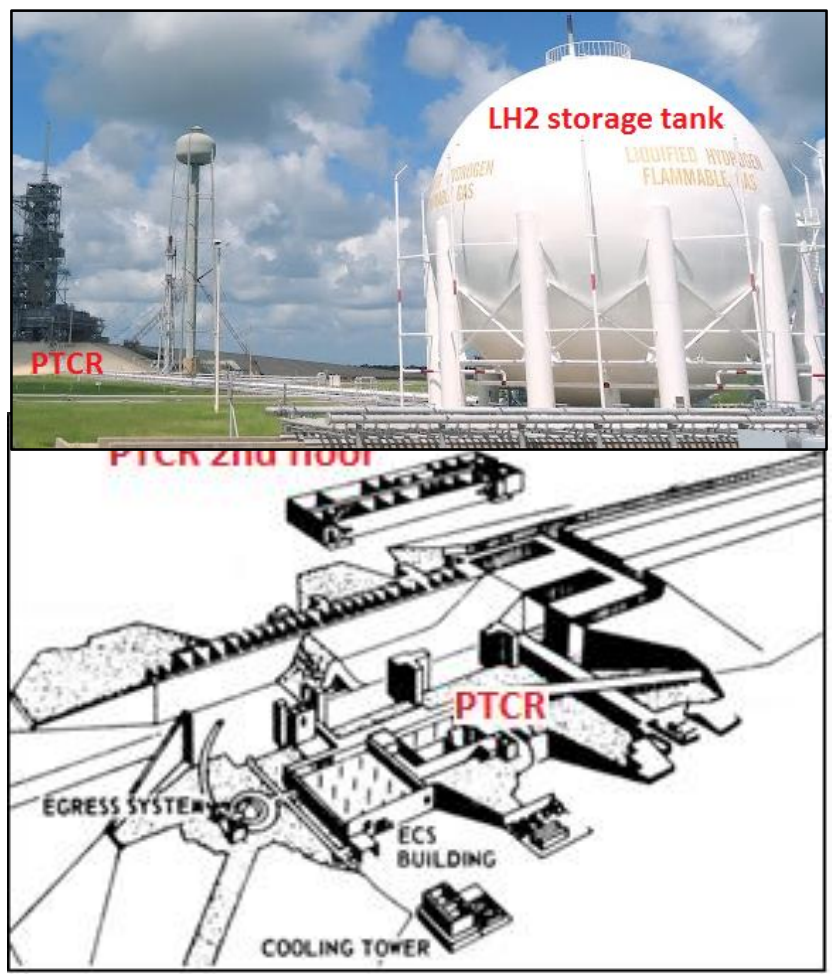

Figure 5. Two PADB channels: $\mathrm{LH}_{2}$ storage tank and PTCR

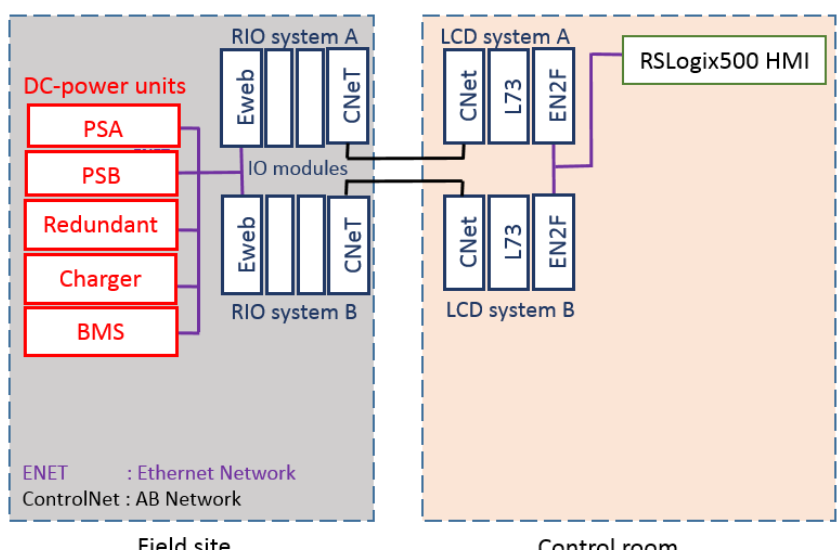

Figure 6. Schematic of a single PADB channel equipped with four VDC-power supply units and one battery-backup unit.

Figure 6 illustrates the schematic of a PADB single channel equipped with four VDC-power supply units and one battery-backup unit located in the field site along with the respective Local Control Distributors (LCD) system located in the control room.

As in ML system, each PADB channel is equipped with four VDC-power supply units (RNDT, CHRG, PSA, PSB), and one Battery unit (BMS). All five units are in concurrent communication through Ethernet Network (ENET) via opensocket data-transfer protocol with two Eweb modules mounted in two separate redundant RIO racks installed along with the IO modules in their respective RIO systems, A and $\mathrm{B}$ as depicted in Figure 6.

Figure 7 illustrates the schematic of all three PADB channels, each channel, equipped with five VDC-power supply units, interconnected via ControlNet Network with LCDs, system A and B. Channels 1 and 2 supply VDC power to the liquid oxygen and liquid hydrogen storage tanks, channel 3 supplies VDC power to PTCR.

Redundant RIO systems A and B independently acquire field data, such as analog signal from sensors and digital signals from indicators, and motorized/manual breakers. Both RIO systems communicate with their respective redundant LCD systems A and B located in the control room via ControlNet ${ }^{\mathrm{TM}}$ network. RNDT, CHRG, and BMS units are in concurrent communication with both redundant Eweb modules mounted in the RIO systems A and B respectively, while PSA and PSB units are in dedicated communication with their respective RIOs (PSA with systems A and PSB with system B).

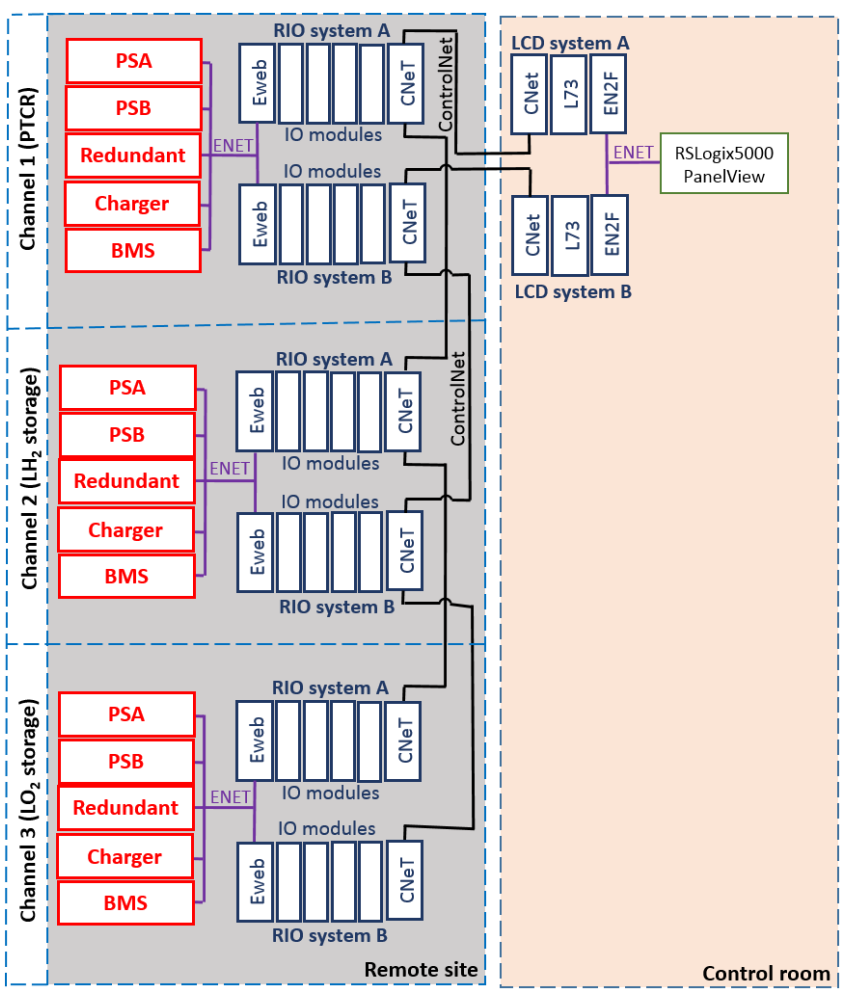

Figure 7. Schematic of all three PADB channels, each channel equipped with four VDC-power supply units and one battery unit.

Four of these five VDC-power units (RNDT, CHRG, PSA, and PSB) depicted in Figure 2 are manufactured by AMETEK, Inc. and have identical specifications. The fifth VDC-power unit, the VDC-battery system or BMS depicted in Figure 3, is manufactured by Navitas Systems, LLC. 
As illustrated in Figure 7 RIO and LCD systems A and B, field RIO, and control room (LCD), are linked via a ControlNet $^{\mathrm{TM}}$ (CNet) communication module installed in their respective racks. LCD systems A and B are linked to the RSLigx5000 Human Machine Interface (HMI) via an Ethernet interface module (EN2F) mounted in the respective racks.

\section{B. PADB SIMULATION REVIEW}

As depicted in Figure 8, emulation of all five VDC-power supply units in a single PADB channel is conducted by five respective Java-based simulation instances that are, as actual VDC-power supply units, in communication with both redundant Eweb modules via ENET and running under a Windows-OS desktop.

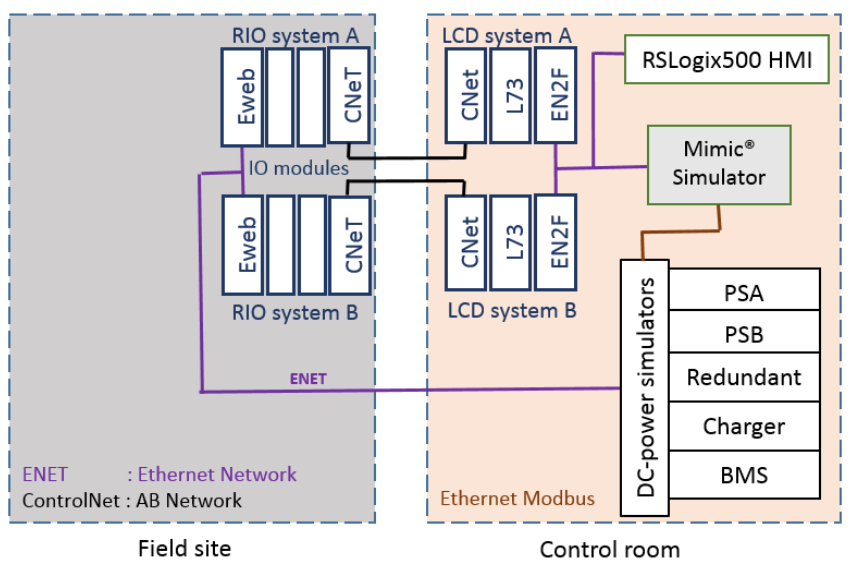

Figure 8. Simulation and emulation of all 5 DC-power units for onechannel GSP system depicted in Figure 5.

Figure 8 depicts Mimic $^{\circledR}$ simulator, also running under Windows OS, and its integration not only with the PLC through LCD and RSLogix5000 HMI via ENET/ControlNet but also with all five Java-based VDC-power supply simulation instances via Modbus.

Four different instances of the same Java software program are launched to simulate and emulate four Ametek VDC-power supplies (RNDT, CHRG, PSA, and PSB), each one having different IP address and port number to communicate via Open-Socket Ethernet architecture with the Eweb modules in system A and B. The simulator emulates and replicates all built-in operative functions in the Ametek VDC-power supply units including trigger and detection of hardware faults.

Figure 2 shows the schematic layout of the multi-thread software program implemented in Java to emulate Ametek VDC-power supplies. The simulated power unit in Java fully emulates the communication protocol of actual Ametek VDC-power supply units. It uses a single port to communicate via open-socket protocol with the Eweb modules in systems A and B, as shown in Figure 2. The multi-thread-based simulator also establishes open-socket communication with the Eweb module just as actual VDC- power supply units do. The simulator launches as many threaded instances as needed, in parallel, to concurrently handle simulations tasks, such as simulator-Eweb data exchange, simulator HMI in Windows OS, VDC-power supply operation, and trigger and detection of hardware faults.

A second Java program was implemented to emulate the BMS unit as its configuration and manufacturer (Navitas) are different from the first four power suppliers. Figure 3 shows the schematic layout of the multi-thread software program using Java to simulate Navitas BMS unit. The BMS unit uses 2 dedicated ports, rather than one (as in the Ametek case) to communicate via open-socket protocol with the Eweb modules in redundant systems A and B. As in the Ametek case, the multi-thread-based simulator also establishes opensocket communication with the Eweb module as actual Navitas BMS unit does. The simulator launches as many threaded instances as needed, in parallel, to concurrently handle simulation tasks, such as simulator-Eweb data exchange, simulator HMI in Windows OS, BMS unit operation, and triggering and detection of hardware faults.

As shown in Figure 7, VDC power is supplied in PADB system through three different channels or sites, PTCR, $\mathrm{LH}_{2}$, and $\mathrm{LO}_{2}$, Figure 9 depicts the comprehensive simulation layout of these three channels.

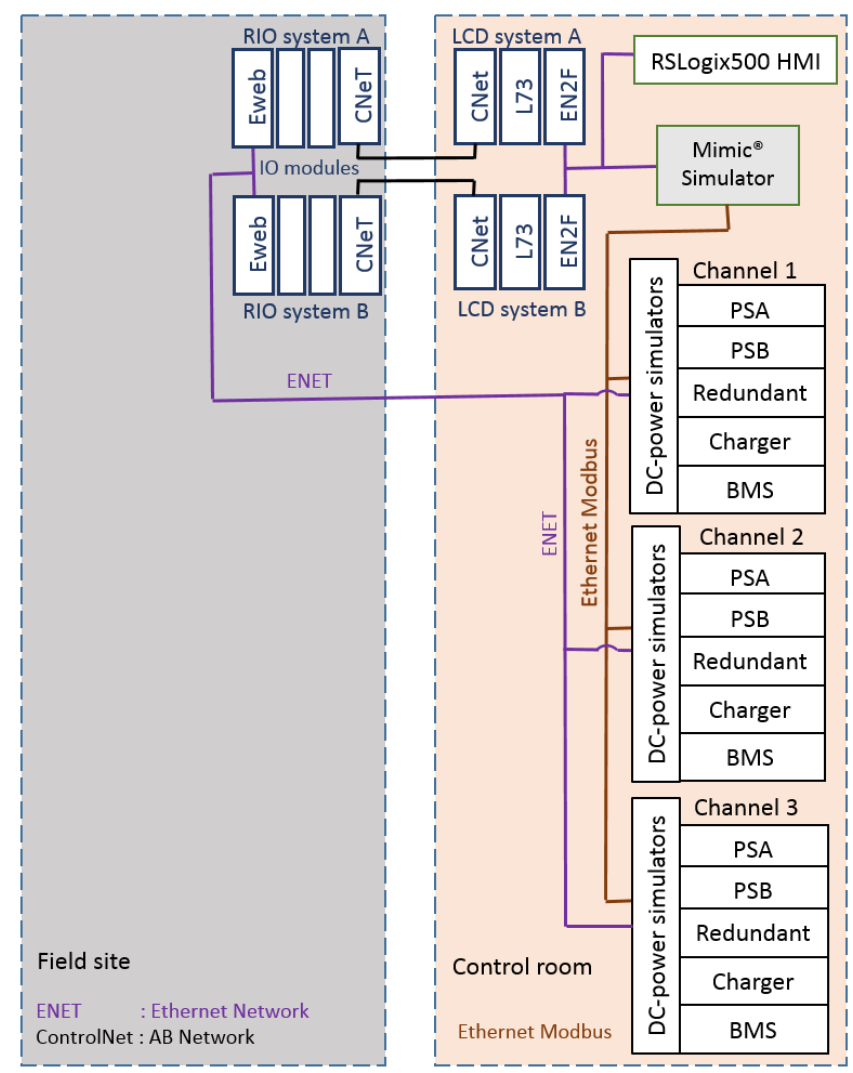

Figure 9. Schematic of simulation on all three PADB channels.

To simulate in parallel all three PADB channels (depicted in Figure 7) it was not necessary to have all three channels 
with their Eweb modules mounted in redundant RIO systems A and B. As shown in Figure 9, only RIO systems A and B of a single channel was used to simulate all three channels as their respective PLC code including Eweb communication were downloaded and run in two redundant RSlogix-5000 controllers mounted in a single-channel LCD systems A and $B$ respectively.

Performance evaluation was also conducted on the RSlogix 5000 controller having two chassis (system A and B) equipped with one Eweb module in each chassis. Accumulative evaluation of communication messages during a week of continuous operation led to zero timeouts and only 9 transmission error were detected. The main objective of this simulation case was to assess the RSlogix 5000 controller on its capacity to handle three channels before having actual VDC-power units for each channel in place.

Figure 10 depicts snapshots of the two coupled HMIs built for PADB simulation running Java-based VDC-power and Mimic $^{\circledR}$-based simulators respectively in parallel in a single Windows-OS environment.
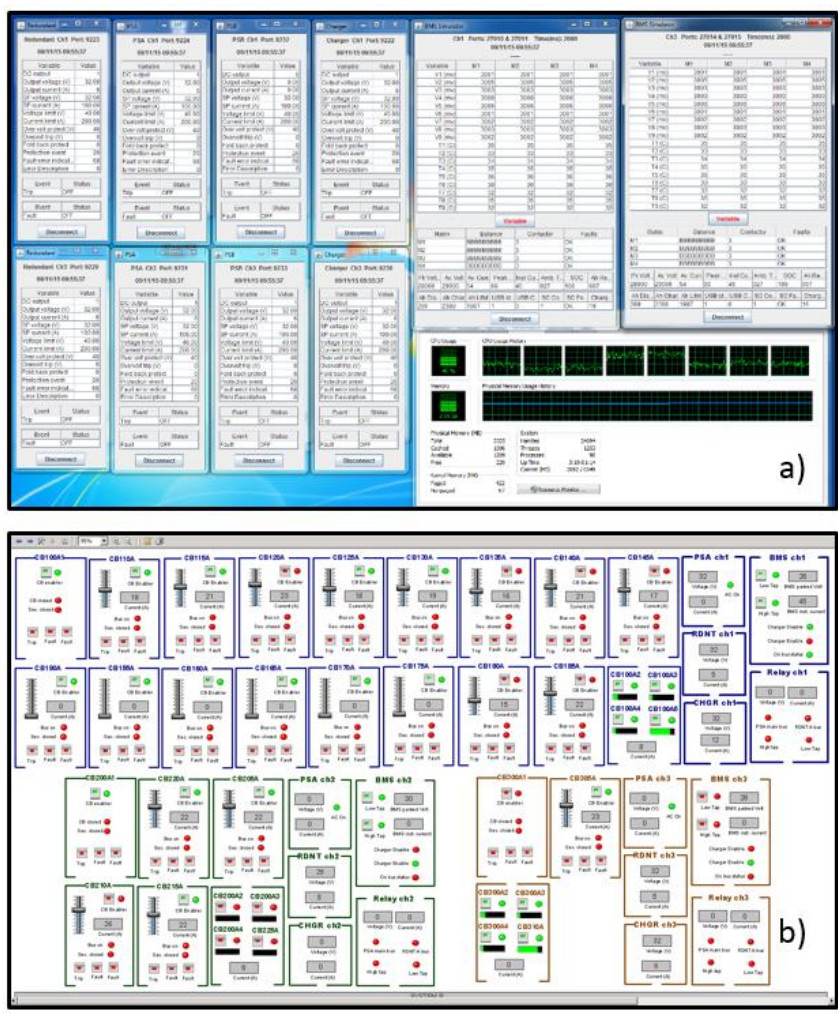

Figure 10. a) Java and b) Mimic ${ }^{\circledR}$ based MHMIs for PADB simulation running concurrently in a Windows-OS desktop.

Figure 10a shows a HMI's snapshot of the Java-based simulation on VDC-power supply units. The snapshot displays two of a total of three PADB channels (HMI for simulated VDC-power supply units in the third channel is displayed in a second screen monitor). Figure $10 \mathrm{~b}$ shows a HMI snapshot of Mimic $^{\circledR}$-based IO simulation of all three channels.
Figure 10a shows simulation of VDC-power supply units in two channels, the four VDC-power units (RNDT, CHRG, PSA, and PSB) of channels 1 are on top left while and the other four units for channel 2 are below on the bottom left. The BMS units of channels 1 and 2 are on the right side of the HMI. The insertion shown on the right bottom of Figure 10a displays RAM memory and CPU usage on running all 15 emulation instances (5 per channel). RAM memory usage happens to be $2.06 \mathrm{~GB}$ from which 1.6 GB are used by Windows OS, meaning that $0.46 \mathrm{~GB}$ are used by the fifteen simulation instances, or approximately $0.03 \mathrm{~GB}(30 \mathrm{MB})$ of RAM per simulation instance.

Figure 10b shows emulation of the IOs in all three channels that includes current loads and their respective motorized and manual circuit breakers. Voltage and current readings acquired from Java-based simulation instances (Figure 10a) are used and displayed on this HMI. Additional operational features, such as low and high tap BMS activation are also emulated through this HMI. Electrical current load value set in this HMI is transferred (via Modbus) to the HMI in Figure 10a to update the current values of the respective VDC-power supply unit.

\section{SUMMARY AND CONCLUSSION}

- Design, development, and deployment of a multi-threaded open-socket parallel simulation and emulation using Java in a Windows OS environment for all VDC-power units used in multichannel GSP applications. Some GSP applications are configured with just one channel (five VDC-power units, and a pair of systems A and B chassis per channel) as in the MPPF. Others, like ML, have as many as four channels (twenty VDC-power units to simulate/emulate concurrently with systems A and B). There is no actual limit to the number of concurrent/parallel VDC-power simulation instances that can simultaneously run. Each simulation instance requires only about $30 \mathrm{MB}$ of RAM.

- Emulation of actual VDC-power units has been achieved with high level of success. All operative functions, such as fault triggering and fault detection have been incorporated in the VDC-power unit simulation instances. TCP Communications via Eweb modules using open-socket method has been replicated to match actual VDC-power units.

- Coupled simulation of digital and analog IOs using Mimic $^{\circledR}$ simulation enabler tool developed by MYNAH Technologies for AB RSLogix5000 controllers. VDCpower loads are coded in a Windows-OS based environment to simulate and emulate rvoltage and current values that accurately represent those in the GSP system main buses.

- As the number of channels increases in present and future GSP applications, simulation/emulation of each of the five VDC-power units per channel becomes crucial to assess 
the performance of the $\mathrm{AB}$ control system without the need to having actual hardware.

- Training operators or changing control strategies on real GSP system can be unsafe or costly. In the virtual GSP site, the control system can be replicated to assess changes on control strategies or conduct training to operators.

- The use of system simulation is also a more cost effective approach for future support of the GSP system. Possible uses for training operators or for development support of future modifications/enhancements to the system can be achieved more efficiently using a virtual GSP system site.

\section{REFERENCES}

[1] Siceloff, Steven (April 12, 2015). "SLS Carries Deep Space Potential".

[2] "NASA finally sets goals, missions for SLS - eyes multistep plan to Mars - NASASpaceFlight.com".

[3] Studio 5000 Logix Designer.

[4] Java NETBEAN IDE

[5] Mimic - Fast, Easy, Flexible Dynamic Simulation for Life-Cycle Results.

[6] AB Eweb: EtherNet/IP Socket Interface

[7] Simply Modbus. 